\title{
Announcement of B. John Davies Prize for the best paper published in IJAMT in 2018
}

\author{
Silvia Schilgerius ${ }^{1}$. Andrew Y. C. Nee ${ }^{2}$ \\ Published online: 11 December 2019 \\ (C) Springer-Verlag London Ltd., part of Springer Nature 2019
}

The B. John Davies Prize for the best paper published in IJAMT 2018 has been awarded to Zhong-Yi Cai, Xiao-Bo Liang, Qing-Min Chen and Xi Zhang. The prize recognizes exceptional articles published in IAMT and awards authors for making an especially significant contribution. The award was named after the late B. John Davies of the University of Manchester Institute of Science and Technology (UMIST), the founding editor-in-chief of IJAMT who led the journal from its launch in 1985 until 2013.

\section{Process}

From all the papers received and published in 2018, five highly rated papers with recommendations by two or more reviewers for the B. John Davies Prize were shortlisted. The shortlisted papers were carefully scrutinized by the regional editors. The following paper has received the highest vote and is recommended for the 2018 B. John Davies Prize.

\section{Title of paper}

Numerical and experimental investigations on the formability of three-dimensional aluminium alloy sandwich panels with egg-box-like cores (The International Journal of Advanced Manufacturing Technology, October 2018, Volume 99, Issue 1-4, pp. 387-397)

\section{Authors}

Zhong-Yi Cai ${ }^{1,2}$, Xiao-Bo Liang ${ }^{2}$, Qing-Min $\mathrm{Chen}^{1}$ and Xi Zhang $^{2}$

\author{
Author affiliations \\ ${ }^{1}$ Roll Forging Research Institute, Jilin University, Changchun, \\ 130025, China \\ ${ }^{2}$ School of Materials Science and Engineering, Jilin \\ University, Changchun 130025, China
}

\begin{abstract}
Light-weight sandwich panels are commonly used in various industries to improve the stiffness and reduce the weight of structure component. For many applications, sandwich panels are required as three-dimensional shells rather than flat plates. However, sandwich structures with curved mid-planes are difficult to make at the present time. This paper was focused on the plastic forming operation of aluminium alloy sandwich plate with egg-box-like (EBL) cores and the possibility to produce a three-dimensional panel from an initially flat sandwich plate was investigated. Detailed numerical simulations have been carried out, the deformation characteristics and the forming defects in the plastic forming process were investigated. The formability of EBL-cored sandwich panel was analyzed, and the limit curve for forming spherical panel was obtained. Multi-point forming experiments were carried out and doubly curved panels with positive and negative Gaussian curvatures were successfully achieved without any forming defect, the satisfactory forming quality of the formed product shapes demonstrated the feasibility of using plastic forming technique to form EBL-cored sandwich panels.
\end{abstract}

Silvia Schilgerius

silvia.schilgerius@ springer.at

Springer Nature, Prinz Eugen-Straße 8-10, 1040 Vienna, Austria

2 Department of Mechanical Engineering, National University of Singapore, 9 Engineering Drive 1, Singapore 117576, Singapore 